\title{
Generalized Exclusion and Hopf Algebras
}

\author{
A. Yildiz \\ Istanbul Technical University, Faculty of Science and Letters, Physics Department, 80626, \\ Maslak, Istanbul, Turkey 円.
}

\begin{abstract}
We propose a generalized oscillator algebra at roots of unity with generalized exclusion and investigate the braided Hopf structure. We find that there are two solutions one of which is the generalized exclusion of the bosonic type and the other is the generalized exclusion of the fermionic type. We also discuss the covariance properties of these oscillators.
\end{abstract}

\section{Introduction}

The generalization of the statistics of identical particles can be done in two different ways in principle. The first approach is based on the generalization of the \pm 1 factor of boson/fermion algebra which comes from the interchange of two identical particles. This approach lead to the introduction of anyons [1] which found applications in fractional quantum Hall effect and superconductivity [2]. The quon algebras [3] and braided oscillators [4] are other generalizations based on the generalizations of the exchange parameter. Mathematically this corresponds to the generalization of the algebra in tensor product space such that

$$
(1 \otimes a)(b \otimes 1)= \pm \pi(a \otimes b)= \pm b \otimes a
$$

(where the + sign refers to bosons and - sign refers to fermions) the permutation map $\pm \pi$ is replaced by a generalized map $\psi$ i.e,

$$
(1 \otimes a)(b \otimes 1)=\psi(a \otimes b) .
$$

In general we have $\psi^{2} \neq i d$ and hence the map $\psi$ is called braiding. The algebra in the tensor product space is connected with Hopf algebras such that the coproduct map $\Delta$ of Hopf algebras is a homomorphism to the tensor product space $\Delta: A \rightarrow A \otimes A$. It creates identical copies of algebras and hence the theory of Hopf algebras can be used in the operator algebra formulations of identical particles. For bosons/fermions ordinary/super Hopf algebras are used and for the generalized exchange phase braided Hopf algebra axioms [5] are used. The braided Hopf algebra axioms are generalizations of ordinary Hopf algebra axioms such that in the limit $\psi \rightarrow \pi$ braided Hopf algebra axioms reduce to the ordinary Hopf algebra axioms.

The other approach for the generalization of statistics is based on the generalization of the Pauli exclusion principle put forward by Haldane [6] and the statistical mechanics is developed by $\mathrm{Wu}[7]$. In the literature an extensive amount of work has been done as applications of this

\footnotetext{
${ }^{1}$ E-mail : yildiz@gursey.gov.tr
} 
generalization. Our aim in this paper is to find an operator algebra for this generalized exclusion. It is known that the $q$-deformed generalization of nilpotent algebras requires the deformation parameter to be a root of unity [12]. For example the q-deformed harmonic oscillators at roots unity is connected with two anyon system 8 . Different versions of the deformation of oscillator algebras and their use in physics can be found in the review [9] and the connection of between q-deformed structures and fractional statistics can be found in [10. In terms of creation and annihilation operators the generalized exclusion can be expressed as $a^{k}=0$ and $\left(a^{\dagger}\right)^{k}=0$. The limit $k \rightarrow 2$ gives the Pauli exclusion principle for fermions. Some operator algebras for fractional exclusion statistics are proposed in ref. [11.

In this work we discuss the problem of the construction of an oscillator algebra unifying the notions of generalized phase (braiding), Hopf algebra and exclusion statistics, i.e., we investigate the braided Hopf algebra structure of a generalized oscillator with generalized exclusion $a^{k}=0$.

\section{Generalized exclusion algebra}

We start with a generalized oscillator algebra generated by $\left(a, a^{*}, q^{N}, q^{-N}, 1\right)$ satisfying

$$
\begin{aligned}
a a^{*}-Q_{1} a^{*} a & =Q_{2} q^{2 N}+Q_{3} q^{-2 N}, \\
a a^{*}-Q_{1}^{-1} a^{*} a & =Q_{2}^{*} q^{-2 N}+Q_{3}^{*} q^{2 N}, \\
a q^{N} & =q q^{N} a, \quad q^{N} a^{*}=q a^{*} q^{N}, \\
a q^{-N} & =q^{-1} q^{-N} a, \quad q^{-N} a^{*}=q^{-1} a^{*} q^{-N} \\
q^{N} q^{-N} & =q^{-N} q^{N}=1_{A}, \quad a^{k}=\left(a^{*}\right)^{k}=0 .
\end{aligned}
$$

where $q$ and $Q_{1}$ are complex parameters of unit modulus and $Q_{2}$ and $Q_{3}$ are any complex parameters and $1_{A}$ is the identity of the algebra. For the ${ }^{*}$-structure we impose

$$
\left(a^{*}\right)^{*}=a, \quad\left(q^{ \pm N}\right)^{*}=q^{\mp N}
$$

From the relations (3) we get

$$
\begin{aligned}
a^{*} a & =\frac{\left(Q_{2}-Q_{3}^{*}\right) q^{2 N}+\left(Q_{3}-Q_{2}^{*}\right) q^{-2 N}}{Q_{1}^{-1}-Q_{1}} \\
a a^{*} & =\frac{\left(Q_{1}^{-1} Q_{2}-Q_{1} Q_{3}^{*}\right) q^{2 N}+\left(Q_{1}^{-1} Q_{3}-Q_{1} Q_{2}^{*}\right) q^{-2 N}}{Q_{1}^{-1}-Q_{1}}
\end{aligned}
$$

We note that in contrast to the operator algebra proposed by Karabali-Nair [1] where the number operator is function of the raising and lowering operators,i.e., $N=f\left(a^{*} a\right)$, we take the Hermitian operator $a^{*} a$ ) as a function of the number operator, i.e., $a^{*} a=f(N)$. Since $a$ and $a^{*}$ are lowering and raising operators respectively the operator $a a^{*}$ should satisfy $a a^{*}=f(N+1)$. The function $f(N)$ for the algebra we propose is given by (5).

$$
q^{2}\left(Q_{2}-Q_{3}^{*}\right)=Q_{1}^{-1} Q_{2}-Q_{1} Q_{3}^{*}
$$

The special cases $Q_{1}=q^{-2}$ and $Q_{1}=q^{2}$, with a rescaling of the generators, reduce to

$$
a a^{*}-q^{-2} a^{*} a=q^{2 N}, \quad a a^{*}-q^{2} a^{*} a=q^{-2 N}
$$


which is the well known q-oscillator algebra [13]. At roots of unity this algebra is related to two anyon system [8]. For $Q_{1} \neq q^{2}, q^{-2}$ we express $Q_{3}$ in terms of $Q_{2}$. Then the oscillator algebra turns out to be

$$
\begin{gathered}
a a^{*}-Q_{1} a^{*} a=Q_{2} q^{2 N}+Q_{1} R Q_{2}^{*} q^{-2 N} \\
a q^{N}=q q^{N} a, \quad q^{N} a^{*}=q a^{*} q^{N}, \quad a^{k}=0
\end{gathered}
$$

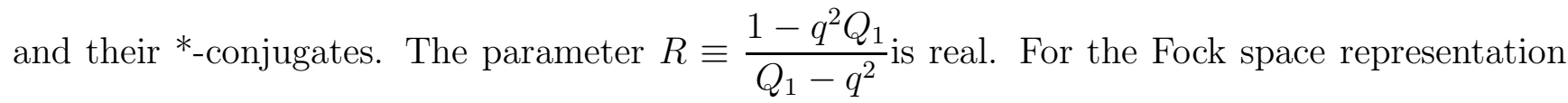
we take

$$
a|n\rangle=a_{n}|n-1\rangle, \quad a^{*}|n\rangle=a_{n+1}^{*}|n+1\rangle, \quad q^{N}|n\rangle=q^{n}|n\rangle .
$$

Solving the difference equations we get the spectrum

$$
a^{*} a|n\rangle=\frac{Q_{2} q^{2 n}-Q_{2}^{*} Q_{1} q^{-2(n-1)}}{q^{2}-Q_{1}}|n\rangle .
$$

The existence of a ground state $a|0\rangle=0$ implies

$$
Q_{2}^{*}=q^{-2} Q_{1}^{-1} Q_{2}
$$

Since $q$ and $Q_{1}$ are complex parameters with unit modulus we write the parameters as

$$
Q_{1}=e^{i \alpha}, \quad q=e^{i \beta}, \quad Q_{2}=B e^{i \gamma}
$$

and substitute these into (11) to get $e^{i 2 \gamma}=e^{i(\alpha+2 \beta)}$. Substituting $Q_{2}$ into the oscillator algebra we get

$$
\begin{array}{r}
a a^{*}-Q_{1} a^{*} a=B q Q_{1}{ }^{1 / 2} q^{2 N}+B q^{-1} Q_{1}^{1 / 2} R q^{-2 N} \\
a a^{*}-Q_{1}^{-1} a^{*} a=B q^{-1} Q_{1}{ }^{-1 / 2} q^{-2 N}+B q Q_{1}^{-1 / 2} R q^{2 N}
\end{array}
$$

Then the spectrum turns out to be

$$
a_{n}^{*} a_{n}=B \frac{\sin (2 n \beta)}{\sin \left(\frac{2 \beta-\alpha}{2}\right)}=B \frac{q^{2 n}-q^{-2 n}}{q Q_{1}^{-1 / 2}-q^{-1} Q_{1}^{1 / 2}} .
$$

The positive definiteness of $a_{n}^{*} a_{n}$ implies that the representation should be finite dimensional with the states $|0\rangle \ldots . .|k-1\rangle$ and the highest state should be annihilated by the raising operator $a^{*}|k-1\rangle=0$. This gives that $q$ is a root of unity $q=e^{i \frac{\pi}{2 k}}$ and taking $\frac{\pi}{k}-2 \pi\left\langle\alpha\left\langle\frac{\pi}{k}\right.\right.$ the spectrum turns out to be

$$
a_{n}^{*} a_{n}=B \frac{\sin \left(n \frac{\pi}{k}\right)}{\sin \frac{1}{2}\left(\frac{\pi}{k}-\alpha\right)} .
$$

Although the deformation parameter $Q_{1}$ (or $\alpha$ ) seems only as a free scaling parameter in the spectrum, its value is going to be fixed by the Hopf algebra structure. 


\section{Braided Hopf Algebra}

The generalization of the permutation map of boson/fermion algebras in tensor product space by a generalized map leads naturally to the generalization of the Hopf algebra called braided Hopf algebra whose axioms in algebraic (not diagrammatic) form read as

$$
\begin{aligned}
m \circ(i d \otimes m) & =m \circ(m \otimes i d) \\
m \circ(i d \otimes \eta) & =m \circ(\eta \otimes i d)=i d \\
(i d \otimes \Delta) \circ \Delta & =(\Delta \otimes i d) \circ \Delta \\
(\epsilon \otimes i d) \circ \Delta & =(i d \otimes \epsilon) \circ \Delta=i d \\
m \circ(i d \otimes S) \circ \Delta & =m \circ(S \otimes i d) \circ \Delta=\eta \circ \epsilon \\
\psi \circ(m \otimes i d) & =(i d \otimes m) \circ(\psi \otimes i d) \circ(i d \otimes \psi) \\
\psi \circ(i d \otimes m) & =(m \otimes i d) \circ(i d \otimes \psi) \circ(\psi \otimes i d) \\
(i d \otimes \Delta) \circ \psi & =(\psi \otimes i d) \circ(i d \otimes \psi) \circ(\Delta \otimes i d) \\
(\Delta \otimes i d) \circ \psi & =(i d \otimes \psi)(\psi \otimes i d) \circ(i d \otimes \Delta) \\
\Delta \circ m & =(m \otimes m)(i d \otimes \psi \otimes i d) \circ(\Delta \otimes \Delta) \\
S \circ m & =m \circ \psi \circ(S \otimes S) \\
\Delta \circ S & =(S \otimes S) \circ \psi \circ \Delta \\
\epsilon \circ m & =\epsilon \otimes \epsilon \\
(\psi \otimes i d) \circ(i d \otimes \psi)(\psi \otimes i d) & =(i d \otimes \psi) \circ(\psi \otimes i d) \circ(i d \otimes \psi)
\end{aligned}
$$

where $m: A \otimes A \rightarrow A$ is the multiplication map, $\Delta: A \rightarrow A \otimes A$ is the comultiplication map, $\eta: K \rightarrow A$ is the unit map, $\epsilon: A \rightarrow K$ is the counit map, $S: A \rightarrow A$ is the antipode map and $\psi: A \otimes A \rightarrow A \otimes A$ is the braiding map. Note that in the limit $\psi \rightarrow \pm \pi$ the braided Hopf algebra axioms reduce to the ordinary/super Hopf algebra axioms. The consistency of the braided Hopf algebra axioms (16) requires that the identity element $1_{A}$ in any algebra should satisfy the following conditions

$$
\Delta\left(1_{A}\right)=1_{A} \otimes 1_{A}, S\left(1_{A}\right)=1_{A}, \epsilon\left(1_{A}\right)=1, \psi\left(1_{A} \otimes a\right)=a \otimes 1_{A}, \psi\left(a \otimes 1_{A}\right)=1_{A} \otimes a \quad \forall a \in A .
$$

The $*$-structure for the braided algebra $B$ satisfies

$$
\begin{aligned}
\Delta \circ * & =\pi \circ(* \otimes *) \circ \Delta \\
S \circ * & =* \circ S .
\end{aligned}
$$

If we interpret the Hopf algebra to define a system (of oscillators e.g.) then the ${ }^{*}$-structure should be defined in such a way that it should be compatible with the algebra in the tensor product space. If $a_{1} \equiv a \otimes 1_{A}$ and $b_{2} \equiv 1_{A} \otimes b$ then

$$
(a \otimes b)^{*}=((a \otimes 1)(1 \otimes b))^{*}=\left(a_{1} b_{2}\right)^{*}=b_{2}^{*} a_{1}^{*}=\psi\left(b^{*} \otimes a^{*}\right)
$$

where in the non-braided limit it gives the ordinary ${ }^{*}$-structure $(a \otimes b)^{*}=a^{*} \otimes b^{*}$. We also note

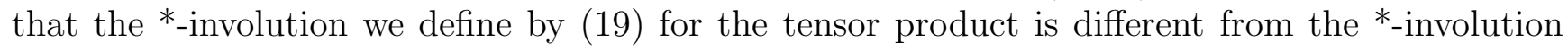
defined by Majid [5]. 
To find the Hopf algebra structure of the oscillator algebra (3) we start with the general forms of the coproducts and braidings which are linear in both of the factors of the tensor product. Although the nonlinear factors are also possible theoretically we do not consider them in this work. Thus we start with the most general linear forms of the coproducts, braidings and antipodes, e.g.,

$$
\begin{gathered}
\Delta\left(q^{N}\right)=D_{1} q^{N} \otimes q^{N}+F_{1} q^{N} \otimes q^{-N}+G_{1} a \otimes a^{*}+H_{1} a^{*} \otimes a+K_{1} 1_{A} \otimes q^{N} \ldots \\
\psi\left(q^{N} \otimes q^{N}\right)=g_{1} q^{N} \otimes q^{N}+g_{2} a \otimes a^{*}+g_{3} a^{*} \otimes a+g_{4} 1_{A} \otimes q^{N}+g_{6} 1_{A} \otimes 1_{A}+\ldots
\end{gathered}
$$

and similar expressions for the other generators of the algebra. It seems that it is very hard to solve the equations for the braided Hopf algebra because there are too many parameters. When we substitute the general forms into the relation $q^{N} q^{-N}=q^{-N} q^{N}=1_{A}$ and use (17) we obtain that the braidings of $q^{N}$ and $q^{-N}$ with other generators are trivial, i.e. they are bosonic elements. From these relations we also obtain that

$$
\Delta\left(q^{N}\right)=D_{1} q^{N} \otimes q^{N}, \quad \Delta\left(q^{-N}\right)=D_{1}^{-1} q^{-N} \otimes q^{-N}, \quad S\left(q^{N}\right)=S_{1} q^{-N}, \quad S\left(q^{-N}\right)=S_{1}^{-1} q^{N}
$$

where $D_{1}$ and $S_{1}$ are complex parameters. This simple form (22) simplifies the solution a lot. Using other relations of the algebra we obtain that the coproducts, the braidings and the antipodes for $a$ and $a^{*}$ are in the form

$$
\begin{array}{r}
\Delta(a)=D_{2} a \otimes q^{N}+D_{3} q^{-N} \otimes a, \quad \Delta\left(a^{*}\right)=D_{3}^{*} a^{*} \otimes q^{N}+D_{2}^{*} q^{-N} \otimes a^{*}, \\
S(a)=-S_{2} a, \quad S\left(a^{*}\right)=-S_{2}^{*} a^{*}, \\
\psi(a \otimes a)=z a \otimes a, \quad \psi\left(a^{*} \otimes a^{*}\right)=z a^{*} \otimes a^{*}, \\
\psi\left(a \otimes a^{*}\right)=z^{-1} a^{*} \otimes a, \quad \psi\left(a^{*} \otimes a\right)=z^{-1} a \otimes a^{*}
\end{array}
$$

where $D_{2}, D_{3}, S_{2}$ are complex parameters and $z$ is a complex parameter of unit modulus.

We substitute these forms into the braided Hopf algebra axioms (16) and find that there are only two solutions one of which is the bosonic generalization of the exclusion with the exchange phase $z=Q_{1}=1$ and the other is the fermionic generalization of the exclusion with the exchange phase $z=Q_{1}=-1$.

\subsection{Generalized exclusion of the bosonic type}

For the first solution we get $Q_{1}=1$, and all braidings turn out to be trivial $(z=1)$. The oscillator algebra

$$
\begin{array}{r}
a a^{*}-a^{*} a=Q_{2}\left(q^{2 N}+q^{-2(N+1)}\right), \\
a q^{N}=q q^{N} a, \quad q^{N} a^{*}=q a^{*} q^{N}, \\
a q^{-N}=q^{-1} q^{-N} a, \quad q^{-N} a^{*}=q^{-1} a^{*} q^{-N} \\
q^{N} q^{-N}=q^{-N} q^{N}=1_{A}, \quad a^{k}=\left(a^{*}\right)^{k}=0
\end{array}
$$

has the Hopf algebra structure

$$
\begin{array}{r}
\Delta\left(q^{N}\right)=D_{1} q^{N} \otimes q^{N}, \Delta\left(q^{-N}\right)=D_{1}^{-1} q^{-N} \otimes q^{-N}, \\
\Delta(a)=D_{2} a \otimes q^{N}+D_{1}^{-2} D_{2} q^{-N} \otimes a
\end{array}
$$




$$
\begin{array}{r}
\Delta\left(a^{*}\right)=D_{1}^{2} D_{2}^{*} a^{*} \otimes q^{N}+D_{2}^{*} q^{-N} \otimes a^{*}, \\
S\left(q^{N}\right)=D_{1}^{-2} q^{-N}, S\left(q^{-N}\right)=D_{1}^{2} q^{N}, \\
S(a)=-q^{-1} a, S\left(a^{*}\right)=-q a^{*} \\
\epsilon\left(q^{N}\right)=D_{1}^{-1}, \epsilon\left(q^{-N}\right)=D_{1}, \epsilon(a)=\epsilon\left(a^{*}\right)=0
\end{array}
$$

where $q=e^{i \frac{\pi}{2 k}}, D_{1}^{4}=-q^{2}, D_{2}^{*} D_{2}=1$ and $Q_{2}=q B$ where $B$ is a free positive parameter. Since all the braidings turn out to be trivial $(\psi=\pi)$ the coproducts, antipodes and counits satisfy the ordinary Hopf algebra axioms. Choosing $B=\frac{1}{2}$ the relation between $a$ and $a^{*}$ can equivalently be written as

$$
a a^{*}-a^{*} a=\cos \frac{(2 N+1) \pi}{2 k}
$$

and the spectrum is given by

$$
a^{*} a|n\rangle=\frac{\sin \frac{n \pi}{k}}{2 \sin \frac{\pi}{2 k}}|n\rangle
$$

This oscillator algebra, in the $k \rightarrow \infty$ limit, reduces to the bosonic oscillator and hence we call it the generalized exclusion algebra of the bosonic type. We note that the exchange phase is constant $(+1)$ and independent of $k$.

\subsection{Generalized exclusion of the fermionic type}

For the second solution we get $Q_{1}=-1$, and the exchange phase for the creation and annihilation operators turn out to be $-1(z=-1)$. The oscillator algebra

$$
\begin{array}{r}
a a^{*}+a^{*} a=Q_{2}\left(q^{2 N}-q^{-2(N+1)}\right), \\
a q^{N}=q q^{N} a, \quad q^{N} a^{*}=q a^{*} q^{N}, \\
a q^{-N}=q^{-1} q^{-N} a, \quad q^{-N} a^{*}=q^{-1} a^{*} q^{-N}, \\
q^{N} q^{-N}=q^{-N} q^{N}=1_{A}, \quad a^{k}=\left(a^{*}\right)^{k}=0
\end{array}
$$

has the Hopf algebra structure

$$
\begin{array}{r}
\Delta\left(q^{N}\right)=D_{1} q^{N} \otimes q^{N}, \quad \Delta\left(q^{-N}\right)=D_{1}^{-1} q^{-N} \otimes q^{-N}, \\
\Delta(a)=D_{2} a \otimes q^{N}+D_{1}^{-2} D_{2} q^{-N} \otimes a, \\
\Delta\left(a^{*}\right)=D_{1}^{2} D_{2}^{*} a^{*} \otimes q^{N}+D_{2}^{*} q^{-N} \otimes a^{*} \\
S\left(q^{N}\right)=q^{-1} q^{-N}, \quad S\left(q^{-N}\right)=q q^{N}, \quad S(a)=-q^{-1} a, \quad S\left(a^{*}\right)=-q a^{*}, \\
\epsilon\left(q^{N}\right)=D_{1}^{-1}, \quad \epsilon\left(q^{-N}\right)=D_{1}, \quad \epsilon(a)=\epsilon\left(a^{*}\right)=0
\end{array}
$$

where $D_{1}^{4}=q^{2}, D_{2}^{*} D_{2}=1, q=e^{i \frac{\pi}{2 k}}$ and $Q_{2}=B q^{1-k}=-B q^{k+1}=-i q B$. The braiding relations are

$$
\psi(a \otimes a)=-a \otimes a, \psi\left(a^{*} \otimes a^{*}\right)=-a^{*} \otimes a^{*}, \psi\left(a \otimes a^{*}\right)=-a^{*} \otimes a, \psi\left(a^{*} \otimes a\right)=-a \otimes a^{*} .
$$

Identifying 


$$
a_{1} \equiv a \otimes 1_{A}, \quad a_{2} \equiv 1_{A} \otimes a, \quad a_{1}^{*} \equiv a^{*} \otimes 1_{A}, \quad a_{2}^{*} \equiv 1_{A} \otimes a^{*}
$$

the braiding relations can equivalently be written as

$$
a_{2} a_{1}=-a_{1} a_{2}, \quad a_{2}^{*} a_{1}^{*}=-a_{1}^{*} a_{2}^{*}, \quad a_{2} a_{1}^{*}=-a_{1}^{*} a_{2}, \quad a_{2}^{*} a_{1}=-a_{1} a_{2}^{*} .
$$

The relation between $a$ and $a^{*}$ can equivalently be written as

$$
a a^{*}+a^{*} a=2 B \sin \frac{(2 N+1) \pi}{2 k}
$$

and the spectrum is given by

$$
a^{*} a|n\rangle=B \frac{\sin \frac{n \pi}{k}}{\cos \frac{\pi}{2 k}}|n\rangle .
$$

Choosing $B=\frac{1}{\sqrt{2}}$ this oscillator gives the usual fermion algebra for $k=2$ and hence we call it the generalized exclusion algebra of the fermionic type. We note that the exchange phase is constant $(-1)$ and independent of $k$.

\section{Covariance}

To study the covariance properties we start with the generalized oscillator algebra (3) and make the transformation

$$
\left(\begin{array}{c}
a \\
a^{*} \\
q^{N} \\
q^{-N}
\end{array}\right)^{\prime}=\left(\begin{array}{cccc}
t_{11} & t_{12} & t_{13} & t_{14} \\
t_{12}^{*} & t_{11}^{*} & t_{14}^{*} & t_{13}^{*} \\
t_{31} & t_{32} & t_{33} & t_{34} \\
t_{32}^{*} & t_{31}^{*} & t_{34}^{*} & t_{33}^{*}
\end{array}\right)\left(\begin{array}{c}
a \\
a^{*} \\
q^{N} \\
q^{-N}
\end{array}\right)
$$

The covariance of $\left(q^{N} q^{-N}=q^{-N} q^{N}=1_{A}\right)^{\prime}$ gives

$$
t_{31}=t_{32}=t_{34}=0, \quad t_{33}^{*} t_{33}=1
$$

and the covariance of $\left(a a^{*}-Q_{1} a^{*} a=Q_{2} q^{2 N}+Q_{3} q^{-2 N}\right)^{\prime}$ gives

$$
\begin{aligned}
t_{11} t_{12}^{*}-Q_{1} t_{12}^{*} t_{11} & =0, \\
t_{12} t_{11}^{*}-Q_{1} t_{11}^{*} t_{12} & =0, \\
Q_{2} t_{11} t_{11}^{*}-Q_{1} Q_{2} t_{12}^{*} t_{12}+t_{13} t_{14}^{*}-Q_{1} t_{14}^{*} t_{13} & =Q_{2} t_{33}^{2}, \\
Q_{3} t_{11} t_{11}^{*}-Q_{1} Q_{3} t_{12}^{*} t_{12}+t_{14} t_{13}^{*}-Q_{1} t_{13}^{*} t_{14} & =Q_{3}\left(t_{33}^{*}\right)^{2}, \\
t_{11} t_{11}^{*}-t_{11}^{*} t_{11}+Q_{1}^{-1} t_{12} t_{12}^{*}-Q_{1} t_{12}^{*} t_{12} & =0 \\
q t_{13} t_{11}^{*}-Q_{1} t_{11}^{*} t_{13}+t_{12} t_{14}^{*}-q Q_{1} t_{14}^{*} t_{12} & =0 \\
q^{-1} t_{11} t_{13}^{*}-Q_{1} t_{13}^{*} t_{11}+t_{14} t_{12}^{*}-q^{-1} Q_{1} t_{12}^{*} t_{14} & =0 \\
q t_{11} t_{14}^{*}-Q_{1} t_{14}^{*} t_{11}+t_{13} t_{12}^{*}-q Q_{1} t_{12}^{*} t_{13} & =0 \\
q^{-1} t_{14} t_{11}^{*}-Q_{1} t_{11}^{*} t_{14}+t_{12} t_{13}^{*}-q^{-1} Q_{1} t_{13}^{*} t_{12} & =0 \\
t_{13} t_{13}^{*}+t_{14} t_{14}^{*}-Q_{1} t_{13}^{*} t_{13}-Q_{1} t_{14}^{*} t_{14} & =0 .
\end{aligned}
$$

The compatibility of these relations with the ${ }^{*}$-operation gives $Q_{1}= \pm 1$ and $Q_{3}=Q_{2}^{*}$. For $Q_{1}=q=1$ the entries of the transformation matrix commute among themselves and setting 
$t_{33}=1$ and $t_{13}=t_{14}=0$ this gives the group $S U(1,1)$. For $q$ a root of unity the covariance of the nilpotency condition $\left(a^{\prime}\right)^{k}=0$ gives $t_{12}=0$. For $Q_{1}=-1$ the last equation in (37) requires $t_{13}=t_{14}=0$ and the transformation for the generalized exclusion of the fermionic type given by (28) turns out to be trivial. The algebra for the generalized exclusion of the bosonic type given by (24) is covariant under the transformation

$$
\left(\begin{array}{c}
a \\
a^{*} \\
q^{N} \\
q^{-N}
\end{array}\right)^{\prime}=\left(\begin{array}{cccc}
t_{11} & 0 & t_{13} & t_{14} \\
0 & t_{11}^{*} & t_{14}^{*} & t_{13}^{*} \\
0 & 0 & t_{33} & 0 \\
0 & 0 & 0 & t_{33}^{*}
\end{array}\right)\left(\begin{array}{c}
a \\
a^{*} \\
q^{N} \\
q^{-N}
\end{array}\right)
$$

if the entries of the transformation matrix satisfy

$$
\begin{aligned}
t_{11} t_{11}^{*} & =t_{11}^{*} t_{11}, \\
t_{11} t_{13} & =q^{3} t_{13} t_{11}, \\
t_{11} t_{13}^{*} & =q t_{13}^{*} t_{11}, \\
t_{11} t_{14} & =q^{-3} t_{14} t_{11}, \\
t_{11} t_{14}^{*} & =q^{-1} t_{14}^{*} t_{11}, \\
t_{11} t_{33} & =t_{33} t_{11}, \\
t_{11} t_{33}^{*} & =t_{33}^{*} t_{11}, \\
t_{13} t_{14} & =q^{-4} t_{14} t_{13}, \\
t_{13} t_{14}^{*}-t_{14}^{*} t_{13}+Q_{2} t_{11} t_{11}^{*} & =Q_{2} t_{33}^{2}, \\
t_{13} t_{13}^{*}-t_{14}^{*} t_{14} & =t_{13}^{*} t_{13}-t_{14} t_{14}^{*}, \\
t_{13} t_{33} & =q t_{33} t_{13}, \\
t_{13} t_{33}^{*} & =q^{-1} t_{33}^{*} t_{13}, \\
t_{14} t_{33} & =q t_{33} t_{14}, \\
t_{14} t_{33}^{*} & =q^{-1} t_{33}^{*} t_{14}, \\
t_{33}^{*} t_{33} & =1, \\
t_{13}^{k} & =t_{14}^{k}=0
\end{aligned}
$$

and $*$-conjugates. The deformation parameter $q$ is a root of unity, $q=e^{i \frac{\pi}{2 k}}$. The coproducts

$$
\begin{array}{ll}
\Delta\left(t_{11}\right)=t_{11} \otimes t_{11}, & \Delta\left(t_{13}\right)=t_{11} \otimes t_{13}+t_{13} \otimes t_{33}, \\
\Delta\left(t_{33}\right)=t_{33} \otimes t_{33}, & \Delta\left(t_{14}\right)=t_{11} \otimes t_{14}+t_{14} \otimes t_{33}^{*}, \\
\Delta\left(t_{11}^{*}\right)=t_{11}^{*} \otimes t_{11}^{*}, & \Delta\left(t_{13}^{*}\right)=t_{11}^{*} \otimes t_{13}^{*}+t_{13}^{*} \otimes t_{33}^{*}, \\
\Delta\left(t_{33}^{*}\right)=t_{33}^{*} \otimes t_{33}^{*}, & \Delta\left(t_{14}^{*}\right)=t_{11}^{*} \otimes t_{14}^{*}+t_{14}^{*} \otimes t_{33},
\end{array}
$$

the antipodes

$$
\begin{array}{r}
S\left(t_{11}\right)=t_{11}^{-1} \quad S\left(t_{13}\right)=-t_{11}^{-1} t_{13} t_{33}^{-1} \quad S\left(t_{14}\right)=-t_{11}^{-1} t_{14} t_{33} \quad S\left(t_{33}\right)=t_{33}^{-1}, \\
S\left(t_{11}^{*}\right)=\left(t_{11}^{*}\right)^{-1}, \quad S\left(t_{13}^{*}\right)=-\left(t_{11}^{*}\right)^{-1} t_{13}^{*} t_{33}, \quad S\left(t_{14}^{*}\right)=-\left(t_{11}^{*}\right)^{-1} t_{14}^{*} t_{33}^{-1}, \quad S\left(t_{33}^{*}\right)=t_{33}
\end{array}
$$

and counits

$$
\begin{gathered}
\epsilon\left(t_{11}\right)=\epsilon\left(t_{11}^{*}\right)=\epsilon\left(t_{33}\right)=\epsilon\left(t_{33}^{*}\right)=1, \\
\epsilon\left(t_{13}\right)=\epsilon\left(t_{13}^{*}\right)=\epsilon\left(t_{14}\right)=\epsilon\left(t_{14}^{*}\right)=0
\end{gathered}
$$


complete the Hopf algebra structure.

\section{Conclusion}

In this work we investigated the braided Hopf algebra structure of generalized oscillator with generalized exclusion $a^{k}=0$, i.e., there can be at most $k-1$ particles at the same state. We find that there are two solutions one of which is the generalized exclusion of the bosonic type where the exchange phase is $(+1)$ and the limit $k \rightarrow \infty$ gives the bosonic oscillator. The second solution is the generalized exclusion of the fermionic type where the exchange phase is $(-1)$ and the limit $k \rightarrow 2$ gives the fermionic oscillator. We note that in the operator algebra with exclusion $a^{k}=0$ we start with the generalized exchange phases (braidings) but the Hopf algebra structure chooses only the \pm 1 factor. If we do not impose any exclusion on the creation-annihilation operators it is possible to find Hopf algebra solutions with the generalized exchange phase (braidings) [4]. Hence, in the context of Hopf algebras, we arrive at the conclusion that the generalization of exclusion does not necessarily imply the generalization of the exchange phase and vice versa.

The Hopf algebras we found for the oscillators can be used to generate the Hopf structure of Lie algebras through a realization using some extension of the Jordan-Schwinger map just like the ones discussed for parabosonic and parafermionic algebras [14].

\section{References}

[1] Wilczek F 1982 Phys. Rev. Lett. 48 1144; Wilczek F 1982 Phys. Rev. Lett. 49957

[2] Wilczek F (ed) 1982 Fractional Statistics and Anyon Superconductivity (Singapore: World Scientific);Chakrabarty T and Pietilainen P (eds) 1988 The Fractional Quantum Hall Effect (Berlin: Springer); Lerda A 1992 Anyons: Quantum Mechanics of Particles with Fractional Statistics (Berlin: Springer)

[3] Greenberg O W 1990 Phys. Rev. Lett. 64 705; Greenberg O W 1991 Phys. Rev. D 43 4111; Mohapatra R N 1990 Phys. Lett. B 242 407; Meljanac S and Perica A 1994 Mod. Phys. Lett. A 9 3293; Marcinek W 1998 Rep. Math. Phys. 41155

[4] Yildiz A 2002 J. Math. Phys. 43 1668; Yildiz A 2002 J. Math. Phys., 43 5459; Baskerville W K and Majid S 1993 J. Math. Phys. 343588

[5] Majid S 1993 Beyond supersymmetry and quantum symmetry (an introduction to braided groups and braided matrices) Quantum Groups, Integrable Statistical Models and Knot Theory edited by Ge M. L. and Vega H. J. (Singapore: World Scientific), 231-282; Majid S 1994 Algebras and Hopf algebras in braided categories Lecture Notes in Pure and Applied Mathematics vol 158 (New York: Marcel Dekker), pp. 55-105 ; Majid S 1995 Foundations of Quantum Group Theory (Cambridge: Cambridge University Press)

[6] Haldane F D M 1991 Phys. Rev.Lett. 67937

[7] Wu Y S 1994 Phys. Rev. Lett.73 922

[8] Floratos E G and Tomaras T N 1990 Phys. Lett. B 251163 
[9] Bonatsos D and Daskolayannis C 1999 Prog. Part. Nucl. Phys. 43537

[10] Chaichian M, Gonzales Felipe R and Montonen C 1993 J. Phys. A: Math. Gen. 264017

[11] Karabali D and Nair V P 1995 Nucl. Phys B 438 [FS] 551; Chen W, Ng Y J and Van Dam H 1996 Mod. Phys. Lett. A 11 795; Speliotopoulos A D 1997 J. Phys.A: Math. Gen. 30 6177; Meljanac S, Milekovic M and Stojic M 1999 J. Phys.A: Math. Gen. 321115

[12] Dunne R S, Macfarlane A J, DeAzcarraga J A and Perez Bueno J C 1997 Int. J. Mod. Phys. A 12 3275; Ahmedov H and Dayi O F 2000 Mod. Phys. Lett. A 15 1801;, Ahmedov H, Yildiz A and Ucan Y 2001 J. Phys. A: Math. Gen. 34, 6413

[13] Biedenharn L C 1989 J. Phys. A: Math. Gen. 22, L873; Macfarlane A J 1989 J. Phys. A: Math. Gen. 22, 4581

[14] Daskaloyannis C, Kanakoglou K and Tsohantjis I 2000 J. Math. Phys. 41652 